\title{
EDITORIAL
}

\section{EVALUACIÓN ECONÓMICA EN SALUD: DE LA INVESTIGACIÓN A LA TOMA DE DECISIONES}

\author{
Juan Rovira \\ Senior Health Economist (Pharmaceuticals) HDNHE, The World Bank
}

La evaluación económica se está consolidando en muchos países como una herramienta para el análisis de las tecnologías y programas sanitarios. Existe un sector importante de empresas consultoras y centros académicos que se dedican de forma exclusiva o preponderante a lo que se conoce como investigación de resultados en salud, que incluye la propia evaluación económica, los estudios de calidad de vida y utilización de tecnologías y otras técnicas que van más allá de la investigación clínica tradicional. Sin embargo, la utilización sistemática, explícita y transparente de la evaluación económica para la toma de decisiones y la asignación de recursos por parte de los entes aseguradores y reguladores del sistema sanitario sólo se da en un número relativamente reducido de países, entre los que destacan el Reino Unido, Australia y Estados Unidos.

España es uno de los países en que, a pesar de que existe la capacidad para llevar a cabo estudios de calidad y un volumen significativo y creciente de trabajos sobre el tema, su aplicación práctica es esporádica y poco conocida.

Correspondencia:

Joan Rovira

Mailstop G7-7021818 H Street

NW Washington DC 20433, USA

Teléfono: 2024584302

Fax: 2025223489
En los párrafos siguientes analizaré algunas de las causas e inconvenientes de esta situación y propondré algunas líneas de avance.

Posiblemente, la causa más importante es la inadecuación de los incentivos que actúan sobre los gestores de los sistemas sanitarios para decidir de forma eficiente. En muchos casos se obtienen mayores ventajas económicas y prestigio utilizando tecnologías nuevas de baja o incierta evidencia que utilizando las opciones más eficientes o coste-efectivas. En la situación habitual de precio nulo para el consumidor de servicios y de retribución no ligada al comportamiento ni al uso de recursos para el profesional, es muy optimista esperar que las consideraciones de coste jueguen un papel importante. La ética médica, la medicina defensiva o el prestigio asociado a la utilización de las novedades tecnológicas son todos ellos factores que a menudo juegan contra el criterio de eficiencia. Los estudios de evaluación económica no son más que información sobre los costes y beneficios potenciales de opciones de decisión, y sólo tendrán un impacto real en la mejora de la eficiencia del sistema sanitario si informan las decisiones de asignación de recursos, pero no si se utiliza sólo para defender o legitimar decisiones tomadas por otras razones.

Pero incluso en los casos en que existe por parte de los gestores una motivación o preo- 
cupación por la eficiencia, existen importantes obstáculos a la utilización de la evaluación económica.

El primero es que la mayor parte de los estudios que se llevan a cabo son realizados o están promocionados por los productores o proveedores de las tecnologías evaluadas, que están obviamente interesados en la difusión y financiación de las mismas por razones comerciales o de otro tipo. Esto hace que muchos gestores supongan, no sin razón, que los resultados de los estudios pueden estar sesgados hacia las opciones que reflejan los intereses del promotor del estudio. A este temor se le añade a menudo una incapacidad práctica para identificar y valorar críticamente y de forma neutral la información disponible.

En este sentido, los estudios constituyen más bien una herramienta de marketing de carácter científico que un instrumento al servicio específico de las personas que toman las decisiones: ciertamente, los estudios pretenden influir en estos últimos, pero no están siempre diseñados y llevados a cabo de acuerdo a sus necesidades específicas. Así, por ejemplo, desde la perspectiva de la salud pública propia del asegurador o regulador, el análisis se debería plantear en términos de qué opción -programa de salud, tecnologíaes la más eficiente para abordar un problema de salud determinado. Para el productor o proveedor el problema es demostrar a los anteriores que su producto o programa es el más eficiente o, por lo menos, razonablemente eficiente. La estrategia analítica habitual es comparar el producto -habitualmente, nuevo-con el más utilizado, es decir, se trata de demostrar la superioridad respecto al «líder» del mercado, aún en el caso de que este último no sea la opción más eficiente. Cabe recordar que los manuales tradicionales recomiendan comparar cualquier opción con todas las técnicamente disponibles para un determinado problema, incluyendo la opción de «no hacer nada». Esta mala práctica respecto a la selección de las opciones comparadas ha sido recientemente criticada en el marco metodológico desarrollado por la Organización Mundial de la Salud (Análisis Coste-Efectividad Generalizado) en el contexto del programa CHOICE. Dicha metodología insiste en que las evaluaciones se lleven a cabo comparando todas las opciones respecto a la opción o estado «nulo», para evitar que la comparación - intencionada o no - respecto a una tecnología ineficiente, haga aparecer «artificialmente» la nueva tecnología como coste-efectiva ${ }^{1}$.

La falta de adecuación de la información sobre coste-efectividad disponible para los gestores no termina aquí. Otras razones son, por ejemplo,

1. La inadecuación del contexto, cuando los estudios están hechos en países con sistemas sanitarios, costes y condiciones de salud sustancialmente distintos a los del entorno del país que toma la decisión.

2. La falta de comparabilidad de los estudios de tecnologías sustitutivas, realizados por distintos analistas sin una metodología estándar.

3. La dificultad de verificar rigurosamente la validez de los estudios publicados, que pocas veces son reproducibles por falta de transparencia del análisis o de imposibilidad de acceso y verificación de los datos originales.

Algunas iniciativas para mejorar la situación y avanzar hacia una mayor y más efectiva utilización de los estudios de evaluación económica pasarían por:

1. Crear una cultura de la eficiencia en el sistema de salud mediante una estrategia integral que incluya formación, información e incentivos apropiados.

2. La iniciativa debería estar liderada por agencias de evaluación de tecnologías

Rev Esp Salud Pública 2004, Vol. 78, N. ${ }^{\circ} 3$ 
con capacidad para identificar las necesidades de los gestores desde una perspectiva de salud pública, establecer prioridades en los temas y tecnologías a evaluar, promover la estandarización y la calidad metodológicas, facilitar la recogida, elaboración y diseminación de información para distintos tipos de gestores, entre otras funciones. Una gran parte de la actividad de las agencias de evaluación -especialmente en países con recursos limitados- debería centrarse en la revisión de la evidencia a partir de la literatura internacional y su adaptación a las condiciones locales.

3. Los gestores comprometidos con la eficiencia y dispuestos a la aplicación de los estudios de evaluación económica deberían hacer los procesos de decisión lo más transparentes posible, indicando los criterios de decisión y la prioridad en la asignación de recursos y el papel que tienen los estudios de evaluación por ejemplo, explicitando qué orden de magnitud de la razón coste-efectividad -el coste por AVAC ganado-se considera razonable para la inclusión de una nueva tecnología entre las que el sistema financia ${ }^{2}$. Esto no implica que se tengan que aplicar dichas reglas de forma automática, sin consideración de otros factores relevantes no considerados en la evaluación económica.
Otro elemento importante sería conseguir un creciente consenso social sobre los requisitos de eficiencia para incorporar y financiar tecnologías y programas, en base a criterios de decisión transparentes, razonables y flexibles, con obvias posibilidades de revisión y actualización en función de la evolución de capacidad económica y las prioridades sociales. Este consenso constituiría a la larga un punto de referencia para las instituciones implicadas en la investigación y desarrollo de nuevas tecnologías, las cuales podrían ajustar adecuadamente sus estrategias empresariales y organizativas a las preferencias sociales.

Sería ingenuo suponer que con ello se lograría eliminar la tensión entre necesidades, desarrollo tecnológico y recursos que caracteriza la mayor parte de las actividades sociales, con un especial dramatismo en el caso de la salud. Pero sí se puede pretender abordar los inevitables conflictos con una mayor racionalidad y transparencia, en beneficio, finalmente, de la eficiencia, la equidad y el bienestar social.

\section{BIBLIOGRAFÍA}

1. Hutubessy R, Chisholm D, Edejer TT. Generalized cost-effectiveness analysis for national-level priority-setting in the health sector. Cost Eff Resour Alloc 2003 Dec 19;1(1):8

2. Ortún V. 30.000 Euros por AVAC. Economía y Salud. Boletín Informativo 2004; núm 49: 1-2. 